\title{
Finite Dimensional Vector Spaces Are Complete for Traced Symmetric Monoidal Categories
}

\author{
Masahito Hasegawa ${ }^{1}$, Martin Hofmann ${ }^{2}$, and Gordon Plotkin ${ }^{3}$ \\ ${ }^{1}$ RIMS, Kyoto University \\ hassei@kurims.kyoto-u.ac.jp \\ ${ }^{2}$ LMU München, Institut für Informatik \\ hofmann@ifi.lmu.de \\ ${ }^{3}$ LFCS, University of Edinburgh \\ gdp@inf.ed.ac.uk
}

\begin{abstract}
We show that the category FinVect F $_{k}$ of finite dimensional vector spaces and linear maps over any field $k$ is (collectively) complete for the traced symmetric monoidal category freely generated from a signature, provided that the field has characteristic 0 ; this means that for any two different arrows in the free traced category there always exists a strong traced functor into FinVect ${ }_{k}$ which distinguishes them. Therefore two arrows in the free traced category are the same if and only if they agree for all interpretations in FinVect $_{k}$.
\end{abstract}

\section{Introduction}

This paper is affectionately dedicated to Professor B. Trakhtenbrot on the occasion of his 85 th birthday. Cyclic networks of various kinds occur in computer science, and other fields, and have long been of interest to Professor Trakhtenbrot: see, e.g., 159168 . In this paper they arise in connection with Joyal, Street and Verity's traced monoidal categories [6]. These categories were introduced to provide a categorical structure for cyclic phenomena arising in various areas of mathematics, in particular knot theory 17]; they are (balanced) monoidal categories [5] enriched with a trace, a natural generalization of the traditional notion of trace in linear algebra that can be thought of as a 'loop' operator.

In computer science, specialized versions of traced monoidal categories naturally arise as recursion/feedback operators as well as cyclic data structures. In particular, Hyland and Hasegawa independently observed a bijective correspondence between Conway (Bekič, or dinatural diagonal) fixpoint operators [11] and traces on categories with finite products 23. Thus, the notion of trace very neatly characterises the well-behaved fixpoint operators commonly used in computer science. More generally, traced symmetric monoidal categories equipped with the additional structure of a cartesian center can be used for modelling recursive computation created from cyclic data structures, see ibid. In this context, freely generated traced symmetric monoidal categories can be characterised as categories of cyclic networks, and so are of particular interest (see 14 for a related treatment).

A. Avron et al. (Eds.): Trakhtenbrot/Festschrift, LNCS 4800, pp. $367+385,2008$.

(C) Springer-Verlag Berlin Heidelberg 2008 
We characterise the equivalence of arrows in free traced symmetric monoidal categories via interpretations in the very familiar setting of linear algebra: the category FinVect Fin $_{k}$ of finite dimensional vector spaces and linear maps over a field $k$. Specifically, we show (Theorem 4) that if $k$ has characteristic 0 then FinVect $_{k}$ is (collectively) complete for the traced symmetric monoidal category freely generated from a signature; this means that for any two different arrows in the free traced category there always exists a structure-preserving functor into FinVect ${ }_{k}$ which distinguishes them. Therefore two arrows in the free traced category are the same if and only if they agree for all interpretations in FinVect F $_{k}$.

In order to show this, we present the freely generated traced symmetric monoidal category in terms of networks modulo suitable isomorphisms, and reduce the problem to that of finding suitable interpretations of these networks in

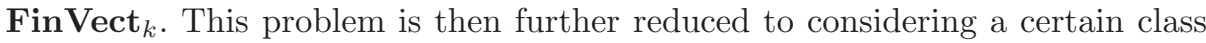
of networks: those over a one-sorted signature and with no inputs or outputs. Finally, given any two such networks $X$ and $Y$, we construct interpretations $\llbracket-\rrbracket^{\mu_{X}}$ and $\llbracket-\rrbracket^{\mu_{Y}}$ such that, ignoring some trivial cases, $\llbracket X \rrbracket^{\mu_{X}}=\llbracket Y \rrbracket^{\mu_{X}}$ and $\llbracket X \rrbracket^{\mu_{Y}}=\llbracket Y \rrbracket^{\mu_{Y}}$ jointly imply that $X$ and $Y$ are isomorphic.

One motivation for our work was previous completeness results for the cartesian case, where the monoidal product is the categorical one. As remarked above, in that case trace operators correspond to Conway fixpoint operators. However, the mathematically natural model categories, such as that of pointed directed complete posets and continuous functions, obey further equations, and the relevant notion is that of an iteration operator [111. It is shown in 11] that any category with an iteration operator satisfying a mild non-triviality condition is collectively complete for the theory of iteration operators. It would be interesting to investigate conditions for the collective completeness of a symmetric monoidal category for trace operators. Another direction which may be of interest would be to look for completeness results for various classes of symmetric monoidal categories equipped with some natural combinations of (co)units and (co)diagonals; see 4] for a discussion of possible such combinations.

A closely related research thread is that of higher-order structures. Concerning coherence problems in category theory, Mac Lane conjectured that the category of vector spaces over a field is complete for the symmetric monoidal closed category freely generated by a set of atoms. This was proved in a more general form by Soloviev [12; his proof-theoretic approach differs substantially from our model-theoretic one. In the cartesian case one considers the typed $\lambda$-calculus, where there is a good deal of work, starting with Friedman's completeness theorem: see [10] and the references given there for further developments. The combination of higher-order structure and traces could be an interesting subject for investigation; specifically one might consider the case of traced symmetric monoidal closed categories.

Organisation of this paper. The rest of this paper is organised as follows. In Sect. 2 we recall the notion of traced symmetric monoidal category, and describe

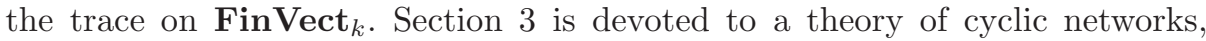
which provide a characterisation of the traced symmetric monoidal category 
freely generated over a monoidal signature. In Sect. 4 we study the interpretation of networks in FinVect , $_{k}$, and, in particular, the interpretations needed for our completeness results. These are presented in the concluding Sect. 5, which also gives a completeness theorem for interpretations with finite fields (Theorem [5), a discussion of some open problems, and a completeness result for compact closed categories (Corollary [5), obtained using the biadjunction of [6] between such categories and traced symmetric monoidal categories.

\section{Preliminaries}

\subsection{Traced Symmetric Monoidal Categories}

A monoidal category is a category $\mathrm{C}$ equipped with a bifunctor $\otimes: \mathrm{C}^{2} \rightarrow \mathrm{C}$, an object $I$ and natural isomorphisms $a_{A, B, C}:(A \otimes B) \otimes C \stackrel{\sim}{\rightarrow} A \otimes(B \otimes C)$, $l_{A}: I \otimes A \stackrel{\sim}{\rightarrow} A$ and $r_{A}: A \otimes I \stackrel{\sim}{\rightarrow} A$ satisfying standard conditions [7]5]. It is strict if these natural isomorphisms are identities. A symmetric monoidal category is a monoidal category equipped with a specified natural isomorphism $c_{X, Y}: X \otimes Y \stackrel{\sim}{\rightarrow} Y \otimes X$, again subject to standard axioms. A trace on such a symmetric monoidal category is a family of functions:

$$
\operatorname{Tr}_{A, B}^{X}: \mathcal{C}(A \otimes X, B \otimes X) \rightarrow \mathcal{C}(A, B)
$$

subject to the following conditions:

- tightening (naturality): $\operatorname{Tr}_{A^{\prime}, B^{\prime}}^{X}\left(\left(k \otimes 1_{X}\right) \circ f \circ\left(h \otimes 1_{X}\right)\right)=k \circ \operatorname{Tr}_{A, B}^{X}(f) \circ h$

- yanking: $\operatorname{Tr}_{X, X}^{X}\left(c_{X, X}\right)=i d_{X}$

- superposition: $\operatorname{Tr}_{C \otimes A, C \otimes B}^{X}\left(i d_{C} \otimes f\right)=i d_{C} \otimes \operatorname{Tr}_{A, B}^{X}(f)$

- exchange:

$$
\operatorname{Tr}_{A, B}^{X}\left(\operatorname{Tr}_{A \otimes X, B \otimes X}^{Y}(f)\right)=\operatorname{Tr}_{A, B}^{Y}\left(\operatorname{Tr}_{A \otimes Y, B \otimes Y}^{X}\left(\left(1_{B} \otimes c_{X, Y}\right) \circ f \circ\left(1_{A} \otimes c_{Y, X}\right)\right)\right)
$$

where, for ease of presentation, the associativity isomorphisms $a$ have been omitted in the last two conditions. For example, the unabbreviated exchange axiom is:

$$
\begin{aligned}
& \operatorname{Tr}_{A, B}^{X}\left(\operatorname{Tr}_{A \otimes X, B \otimes X}^{Y}(f)\right)= \\
& \operatorname{Tr}_{A, B}^{Y}\left(\operatorname{Tr}_{A \otimes Y, B \otimes Y}^{X}(\right. \\
& \left.\left.\quad a_{B, Y, X}^{-1} \circ\left(1_{B} \otimes c_{X, Y}\right) \circ a_{B, X, Y} \circ f \circ a_{A, X, Y}^{-1} \circ\left(1_{A} \otimes c_{Y, X}\right) \circ a_{A, Y, X}\right)\right)
\end{aligned}
$$

where $f:(A \otimes X) \otimes Y \rightarrow(B \otimes X) \otimes Y$. Note that this axiomatisation is not quite the same as the original axiomatisation [6] or another popular formulation (see e.g., [23]); however, it is not hard to see that they are all equivalent $1 \mathrm{~A}$ traced symmetric monoidal category is a symmetric monoidal category equipped with a (specified) trace.

The following graphical notation for the trace may help the reader. Given $f: A \otimes X \rightarrow B \otimes X$, its trace $\operatorname{Tr}_{A, B}^{X}(f): A \rightarrow B$ is shown as a feedback:

${ }^{1}$ The vanishing condition for the unit $\operatorname{Tr}^{I}(f)=f$ was redundant in the original axiomatisation. The vanishing condition for tensor $\operatorname{Tr}^{X \otimes Y}(f)=\operatorname{Tr}^{X}\left(\operatorname{Tr}^{Y}(f)\right)$ and the sliding condition $\operatorname{Tr}^{X}((1 \otimes h) \circ f)=\operatorname{Tr}^{Y}(f \circ(1 \otimes h))$ can all be derived from the axioms presented here. 
tightening : $\operatorname{Tr}_{A^{\prime}, B^{\prime}}^{X}\left(\left(k \otimes 1_{X}\right) \circ f \circ\left(h \otimes 1_{X}\right)\right)=k \circ \operatorname{Tr}_{A, B}^{X}(f) \circ h$

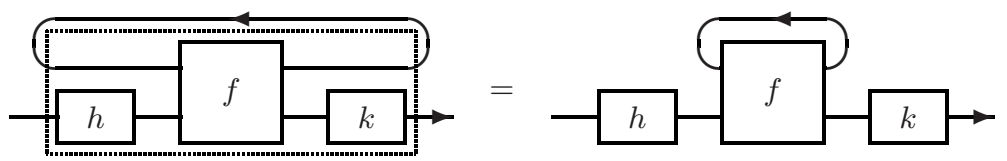

yanking : $\operatorname{Tr}_{X, X}^{X}\left(c_{X, X}\right)=1_{X}$

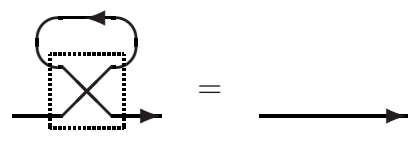

superposition : $\operatorname{Tr}_{C \otimes A, C \otimes B}^{X}\left(1_{C} \otimes f\right)=1_{C} \otimes \operatorname{Tr}_{A, B}^{X}(f)$

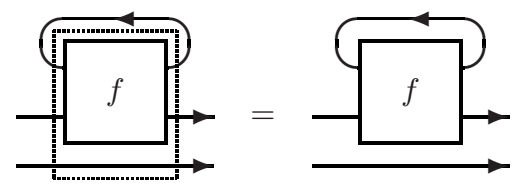

exchange : $\operatorname{Tr}_{A, B}^{X}\left(\operatorname{Tr}_{A \otimes X, B \otimes X}^{Y}(f)\right)=\operatorname{Tr}_{A, B}^{Y}\left(\operatorname{Tr}_{A \otimes Y, B \otimes Y}^{X}\left(\left(1_{B} \otimes c_{X, Y}\right) \circ f \circ\left(1_{A} \otimes c_{Y, X}\right)\right)\right)$

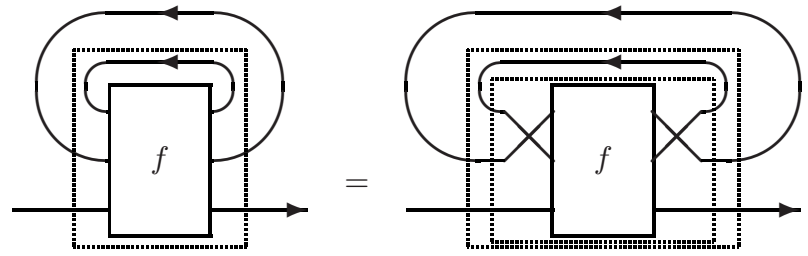

Fig. 1. Axioms for Trace

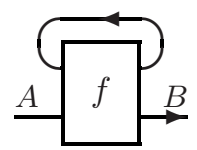

The above axioms are presented using this notation in Figure 1

\subsection{Finite Dimensional Vector Spaces}

Finite dimensional vector spaces over a field $k$ and linear maps form a traced symmetric monoidal category FinVect ${ }_{k}$. The monoidal structure is given by the standard tensor product, and the trace is a natural generalization of the standard 'sum of diagonal elements' trace, sometimes called the 'partial trace'; the trace $\operatorname{Tr}_{U, V}^{W}(f): U \rightarrow V$ of a linear map $f: U \otimes_{k} W \rightarrow V \otimes_{k} W$ is given by:

$$
\left(\operatorname{Tr}_{U, V}^{W}(f)\right)_{i, j}=\Sigma_{k} f_{i \otimes k, j \otimes k}
$$

where $i, j$ run over bases of $U$ and $V$. If $U=V=k$, we have $\operatorname{Tr}^{W}(f)=\sum_{k} f_{k, k}$ as expected. If $\left\{e_{1}, \ldots, e_{n}\right\}$ is a basis of $W$, this is the same as $\sum_{i}\left\langle f\left(e_{i}\right) \mid e_{i}\right\rangle$ where $\langle-\mid-\rangle$ is the canonical scalar product such that $\left\langle e_{i} \mid e_{j}\right\rangle=\delta_{i j}$. 
The partial trace is the unique trace for this monoidal structure on FinVect . $_{\text {. }}$

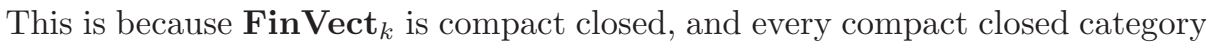
has a unique trace with respect to its monoidal structure.

\section{Cyclic Networks}

We present a theory of cyclic networks similar to the theory of cyclic sharing graphs given in [3].

\subsection{Sorts and Signatures}

We introduce a notion of multisorted signature suitable for interpretation over monoidal categories. If $S$ is our set of sorts we call elements of $S^{*}$, the set of finite sequences of sorts, arities. Given such an arity $v$, we write $|v|$ for its length and $v_{i}$ for its $i$-th component (for $1 \leq i \leq|v|$ ).

Definition 1. An $S$-sorted signature is a triple $\left(F, a r_{i n}, a r_{\text {out }}\right)$ where $F$ is a set whose elements are called function symbols, and where $a r_{i n}, a r_{\text {out }}: F \rightarrow S^{*}$ are mappings assigning to each function symbol $f$ two arities: an input arity $a r_{i n}(f)$ and an output arity $a r_{\text {out }}(f)$.

We may refer to a signature by the set $F$ alone, leaving the arity functions implicit.

Definition 2. We define $F_{\bullet}$ to be the extension of $F$ with additional function symbols $\bullet_{s}$ for each sort $s \in S$, with $\operatorname{ar}_{\text {in }}\left(\bullet_{s}\right)=\operatorname{ar}_{\text {out }}\left(\bullet_{s}\right)=\varepsilon$.

The function symbol $\bullet s$ will be used to represent the trivial cycle of sort $s$ (the trace of the identity at $s$ ).

\subsection{Networks}

Definition 3. Let $F$ be an $S$-sorted signature. A network from $v$ to $w$ in $S^{*}$ over $F$ is a tuple $N$ of the form $(X, \varphi, \pi)$, where:

- $X$ is a finite set (of nodes)

$-\varphi$ is a function from $X$ to $F_{\bullet}$ (the labelling function, assigning a function symbol to each node)

$-\pi$ is a bijection between

$$
O_{N}=\left\{\langle x, i\rangle|x \in X, 1 \leq i \leq| \operatorname{ar}_{\text {out }}(\varphi(x)) \mid\right\} \cup\{j|1 \leq j \leq| v \mid\}
$$

and

$$
D_{N}=\left\{\langle x, i\rangle|x \in X, 1 \leq i \leq| \operatorname{ar}_{i n}(\varphi(x)) \mid\right\} \cup\{j|1 \leq j \leq| w \mid\}
$$


such that the following constraints on arities are satisfied:

$-\pi\langle x, i\rangle=\langle y, j\rangle$ implies $\operatorname{ar}_{\text {out }}(\varphi(x))_{i}=\operatorname{ar}_{\text {in }}(\varphi(y))_{j}$

$-\pi\langle x, i\rangle=j$ implies ar out $_{\text {ou }}(\varphi(x))_{i}=w_{j}$

$-\pi(i)=\langle y, j\rangle$ implies $v_{i}=\operatorname{ar}_{i n}(\varphi(y))_{j}$

$-\pi(i)=j$ implies $v_{i}=w_{j}$

We say that $v$ and $w$ are the input and output arities of the network, and write $N: v \rightarrow w$.

It may help the reader to think of $O$ as the set of ports from which flow originates and $D$ as the set of ports to which flow goes. The function $\pi$ then shows how the ports are linked.

Example 1. Let $S=\{\mathrm{A}, \mathrm{B}\}$ be the set of sorts. We consider the following signature $\left(F, \operatorname{ar}_{\text {in }}, \operatorname{ar}_{\text {out }}\right)$ on $S$, where $F=\{\mathrm{f}, \mathrm{g}\}$ and:

$$
\begin{aligned}
\operatorname{ar}_{\text {in }}(\mathrm{f}) & =\mathrm{AB} \operatorname{ar}_{\text {out }}(\mathrm{f})=\mathrm{AA} \\
\operatorname{ar}_{\text {in }}(\mathrm{g}) & =\mathrm{A} \quad \operatorname{ar}_{\text {out }}(\mathrm{g})=\mathrm{B}
\end{aligned}
$$
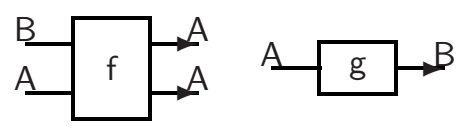

Then, for instance, $(\{f, g, a\}, \varphi, \pi): \mathrm{A} \rightarrow \mathrm{A}$ with $\varphi(f)=\mathrm{f}, \varphi(g)=\mathrm{g}, \varphi(a)=\bullet \mathrm{A}$ and:

$$
\begin{aligned}
& \pi\langle f, 1\rangle=1 \\
& \pi\langle f, 2\rangle=\langle g, 1\rangle \\
& \pi\langle g, 1\rangle=\langle f, 2\rangle \\
& \pi(1)=\langle f, 1\rangle
\end{aligned}
$$

is a network which may be pictured as follows:

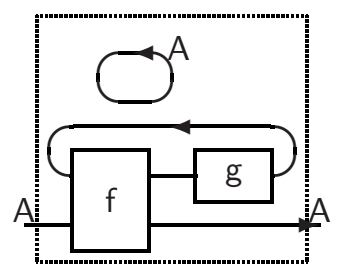

\subsection{Homomorphisms}

Definition 4. Let $N=(X, \varphi, \pi): v \rightarrow w$ and $N^{\prime}=\left(X^{\prime}, \varphi^{\prime}, \pi^{\prime}\right): v \rightarrow w$ be networks with the same input and output arities. A homomorphism from $N$ to $N^{\prime}$ is given by a function $f: X \rightarrow X^{\prime}$ such that:

$-\varphi^{\prime}(f(x))=\varphi(x)$

- $\pi\langle x, i\rangle=\langle y, j\rangle$ implies $\pi^{\prime}\langle f(x), i\rangle=\langle f(y), j\rangle$

- $\pi\langle x, i\rangle=j$ implies $\pi^{\prime}\langle f(x), i\rangle=j$

- $\pi(i)=\langle y, j\rangle$ implies $\pi^{\prime}(i)=\langle f(y), j\rangle$

- $\pi(i)=j$ implies $\pi^{\prime}(i)=j$ 
The first condition just says that $f$ does not change the function symbol assigned to each node. The other four requirements are equivalent to the commutation of the following diagram:

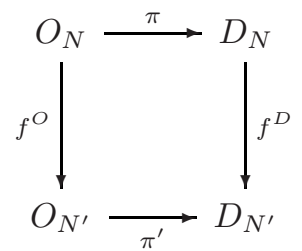

where $f^{O}$ and $f^{D}$ send $\langle x, i\rangle$ to $\langle f(x), i\rangle$ and $j$ to $j$.

We evidently have a category with objects the networks of given input and output arities and morphisms the homomorphisms. Since, as one easily sees, the inverse of a bijective homomorphism is also a homomorphism, the isomorphisms are the bijective homomorphisms. Note that we deal with trivial cycles as nodes and hence homomorphisms must send trivial cycles to trivial cycles.

\subsection{Interpretations in Traced Categories}

Let us fix a traced symmetric monoidal category $\mathcal{C}$. We are mainly interested in the case of finite dimensional vector spaces and linear maps over a field, but it is natural to state the general case, and necessary if we want to say something about the classifying category built from networks.

Definition 5. Let $F$ be an $S$-sorted signature. Then an interpretation $\mu$ of $F$ in $\mathrm{C}$ consists of the following data:

- an object $\llbracket s \rrbracket^{\mu}$ of $\mathcal{C}$ for each sort $s \in S$

- an arrow $\llbracket f \rrbracket^{\mu}: \llbracket a r_{i n}(f) \rrbracket^{\mu} \rightarrow \llbracket \operatorname{ar}_{\text {out }}(f) \rrbracket^{\mu}$ for each function symbol $f \in F$, while for $\bullet_{s}$ we put $\llbracket \bullet_{s} \rrbracket^{\mu}=\operatorname{Tr} \llbracket \rrbracket^{\mu}\left(i d_{\llbracket s \rrbracket^{\mu}}\right)$

where we define the interpretation of arities by $\llbracket \varepsilon \rrbracket^{\mu}=I$ and $\llbracket s w \rrbracket^{\mu}=\llbracket s \rrbracket^{\mu} \otimes \llbracket w \rrbracket^{\mu}$.

Definition 6. Let $F$ be an $S$-sorted signature and let $\mu$ be an interpretation of $F$. Then the value $\llbracket(X, \varphi, \pi) \rrbracket^{\mu}: \llbracket v \rrbracket^{\mu} \rightarrow \llbracket w \rrbracket^{\mu}$ of a network $(X, \varphi, \pi): v \rightarrow w$ with respect to $\mu$ is defined to be the trace of:

$$
\begin{aligned}
& \left(\bigotimes_{x \in X} \llbracket \operatorname{ar}_{\text {out }}(\varphi(x)) \rrbracket^{\mu}\right) \otimes \llbracket v \rrbracket^{\mu} \stackrel{\hat{\pi}}{\rightarrow}\left(\bigotimes_{x \in X} \llbracket a r_{\text {in }}(\varphi(x)) \rrbracket^{\mu}\right) \otimes \llbracket w \rrbracket^{\mu} \\
& \stackrel{\left(\otimes \llbracket \varphi(x) \rrbracket^{\mu}\right) \otimes \llbracket w \rrbracket^{\mu}}{\longrightarrow}\left(\bigotimes_{x \in X} \llbracket a r_{\text {out }}(\varphi(x)) \rrbracket^{\mu}\right) \otimes \llbracket w \rrbracket^{\mu}
\end{aligned}
$$

where $\hat{\pi}$ is the isomorphism induced by $\pi$.

Proposition 1. If two networks are isomorphic, they have the same value.

\subsection{The Traced Monoidal Category of Networks}

Fixing an $S$-sorted signature $F$, we now define several constructions on networks over $F$. 
Definition 7. - Identity Networks. The identity network on arity $v$ is defined to be $(\emptyset, \emptyset, i d): v \rightarrow v$, where id is the identity permutation.

- Sequential Composition of Networks. For networks $N=(X, \varphi, \pi): v \rightarrow w$ and $N^{\prime}=\left(X^{\prime}, \varphi^{\prime}, \pi^{\prime}\right): w \rightarrow u$, their sequential composition $N^{\prime} \circ N: v \rightarrow u$ is the network $\left(X \uplus X^{\prime}, \varphi \uplus \varphi^{\prime}, \pi^{\prime \prime}\right): v \rightarrow u$, where $\left(\varphi \uplus \varphi^{\prime}\right)(x)=\varphi(x)$ for $x \in X$ and $\left(\varphi \uplus \varphi^{\prime}\right)(y)=\varphi^{\prime}(y)$ for $y \in X^{\prime}$, and $\pi^{\prime \prime}$ sends (i) $p \in O_{N}$ to $\pi^{\prime}(\pi(p))$ if $\pi(p) \in \mathbb{N}$, otherwise to $\pi(p)$, and (ii) $\langle y, j\rangle \in O_{N^{\prime}}$ to $\pi^{\prime}\langle y, j\rangle$.

- Parallel Composition of Networks. For networks $N=(X, \varphi, \pi): v \rightarrow w$ and $N^{\prime}=\left(X^{\prime}, \varphi^{\prime}, \pi^{\prime}\right): v^{\prime} \rightarrow w^{\prime}$, their parallel composition $N \otimes N^{\prime}: v v^{\prime} \rightarrow w w^{\prime}$ is the network $\left(X \uplus X^{\prime}, \varphi \uplus \varphi^{\prime}, \pi^{\prime \prime}\right): v v^{\prime} \rightarrow w w^{\prime}$ where $(i) \pi^{\prime \prime}(p)=\pi(p)$ for $p \in O_{N}$, (ii) $\pi^{\prime \prime}(|v|+i)=|w|+\pi^{\prime}(i) \quad\left(1 \leq i \leq\left|v^{\prime}\right|\right)$ if $\pi^{\prime}(i) \in \mathbb{N}$, otherwise $\pi^{\prime \prime}(|v|+i)=\pi^{\prime}(i)$, and (iii) $\pi^{\prime \prime}\langle y, i\rangle=|w|+\pi^{\prime}\langle y, i\rangle$ if $\pi^{\prime}\langle y, i\rangle \in \mathbb{N}$, otherwise $\pi^{\prime \prime}\langle y, i\rangle=\pi^{\prime}\langle y, i\rangle$.

- Symmetry Networks. The symmetry network on arities $v$ and $w$ is defined to be $\left(\emptyset, \emptyset, c_{|v|,|w|}\right): v w \rightarrow$ wv where $c_{m, n}(i)=i+n$ for $1 \leq i \leq m$ and $c_{m, n}(m+i)=i$ for $1 \leq i \leq n$.

- Traces of Networks. The trace $\operatorname{Tr}_{v, w}^{s}(N): v \rightarrow w$ of $N=(X, \varphi, \pi): v s \rightarrow w s$ is the network:

- $\left(X \uplus\{a\}, \varphi^{\prime}, \pi^{\prime}\right): v \rightarrow w$ if $\pi(|v|+1)=|w|+1$, where $\varphi^{\prime}(x)=\varphi(x)$ for $x \in X$ and $\varphi^{\prime}(a)=\bullet_{s}$, and $\pi^{\prime}=\pi \backslash\{\langle|v|+1,|w|+1\rangle\}$.

- $\left(X, \varphi, \pi^{\prime}\right): v \rightarrow w$ if $\pi(|v|+1) \neq|w|+1$, where $\pi^{\prime}(p)=\pi(|v|+1)$ if $p=\pi^{-1}(|w|+1)$ and $\pi^{\prime}(p)=\pi(p)$, otherwise..

This definition is extended to non-primitive arities by setting $\operatorname{Tr}_{v, w}^{\varepsilon}(N)=N$ for $N: v \rightarrow w$ and $\operatorname{Tr}_{v, w}^{s u}(N)=\operatorname{Tr}_{v, w}^{s}\left(\operatorname{Tr}_{v s, w s}^{u}(N)\right)$ for $N: v s u \rightarrow$ wsu.

Lemma 1. The constructions above are well-defined on equivalence classes of networks up to network isomorphism.

We can now introduce the traced symmetric monoidal category $\operatorname{Net}_{(S, F)}$. Its objects are the arities (elements of $S^{*}$ ) and an arrow from $v$ to $w$ is an equivalence class of networks over $F$ with input arity $v$ and output arity $w$, up to network isomorphism. Composition is given by sequential composition, and the identity arrows by the identity networks. The tensor of two objects is their concatenation and the tensor of two arrows is given by parallel composition; the symmetry maps are given by the symmetry networks. Finally, trace is given by the trace on networks. Using the above lemma it is now straightforward to show:

Proposition 2. $\boldsymbol{N e t}_{(S, F)}$ forms a traced strict symmetric monoidal category.

\section{6 $\operatorname{Net}_{(S, F)}$ as a Classifying Category}

Just as in traditional functorial model theory, it is not hard to see that giving an interpretation of an $S$-sorted signature $F$ in a traced symmetric monoidal category $\mathcal{C}$ is equivalent to giving a structure-preserving functor (traced functor) from $\operatorname{Net}_{(S, F)}$ to $\mathcal{C}$. This observation can be strengthened to be an equivalence of the category $\operatorname{Mod}((S, F), \mathcal{C})$ of interpretations of $F$ in $\mathcal{C}$ and the category 
$\operatorname{TrMon}\left(\operatorname{Net}_{(S, F)}, \mathcal{C}\right)$ of traced functors from $\operatorname{Net}_{(S, F)}$ to $\mathcal{C}$ and monoidal natural transformations, where we define a morphism between interpretations $\mu$ and $\mu^{\prime}$ to be a family of arrows $h_{s}: \llbracket s \rrbracket^{\mu} \rightarrow \llbracket s \rrbracket^{\mu^{\prime}}$ which commutes with the interpretations of function symbols, that is, for $f$ with $a r_{i n}(f)=s_{1} \ldots s_{m}$ and $a r_{\text {out }}(f)=$ $t_{1} \ldots t_{n}$, the following diagram commutes:

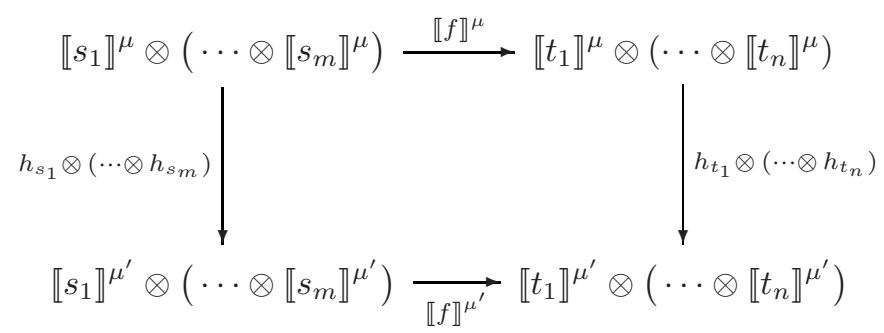

Proposition 3. There is an equivalence of categories:

$$
\operatorname{Mod}((S, F), \mathcal{C}) \simeq \operatorname{TrMon}\left(\operatorname{Net}_{(S, F)}, \mathcal{C}\right)
$$

Proof (Outline). Given an interpretation in a traced (possibly non-strict) symmetric monoidal category $\mathcal{C}$, we can extend it to a strong traced functor from $\operatorname{Net}_{(S, F)}$ to $\mathcal{C}$. This also sends morphisms between interpretations to monoidal natural transformations, and we obtain a fully faithful functor from Mod $((S, F), \mathcal{C})$ to $\operatorname{TrMon}\left(\operatorname{Net}_{(S, F)}, \mathcal{C}\right)$. In addition, given a strong traced functor from $\operatorname{Net}_{(S, F)}$, we can construct an isomorphic strong traced functor which comes from an interpretation.

\section{Networks, Homomorphisms and Interpretations in Finite Dimensional Vector Spaces}

We have seen that to give a strict traced functor from $\mathbf{N e t}_{(S, F)}$ to a traced symmetric monoidal category $\mathcal{C}$ is to give an interpretation of the signature $(S, F)$ in $\mathcal{C}$. We are particularly interested in interpretations in FinVect ${ }_{k}$, for various fields $k$; we call such interpretations interpretations over $k$. Proposition 1 gives us the soundness of such interpretations:

If two networks are isomorphic, they have the same value for all interpretations over any field $k$.

Our aim is to establish the converse when $k$ has characteristic 0 :

If two networks have the same value under all interpretations over $k$ then they are isomorphic.

To this end a number of simplifying assumptions will prove convenient:

- We consider only the single-sorted case. This will involve no loss of generality, due to the following: any signature $F$ has an associated single-sorted signature $F_{o}$ obtained by identifying all its sorts; any network $N$ over $F$ then has 
an associated network $N_{o}$ over $F_{o}$; and for any networks $N, N^{\prime}: u \rightarrow v$ over $F$, if $N_{o}$ and $N_{o}^{\prime}$ are isomorphic, so are $N$ and $N^{\prime}$. In the single-sorted case we identify arities with non-negative integers and write $\bullet$ for the (unique) function symbol for trivial cycles.

- In the single-sorted case, we consider only closed networks, those with no inputs and outputs and so of the form $N: 0 \rightarrow 0$. We will later reduce the case of non-closed networks to that of closed ones: introducing extra (dummy) function symbols $f_{m}: 0 \rightarrow m$ and $f^{n}: n \rightarrow 0$ for all $m, n>0$, one has that two networks $N, N^{\prime}: m \rightarrow n$ are isomorphic if and only if their compositions with (the networks consisting of) $f_{m}$ and $f^{n}$ are isomorphic.

- Finally, we consider only non-empty networks without trivial cycles, i.e., those which do not contain any $\bullet$-labelled node. The more general case will not present significant additional difficulties.

So, in the rest of this section, by a network we mean, unless otherwise stated, a non-empty closed network without trivial cycles over a single-sorted signature.

\subsection{Basic Facts about Networks and Homomorphisms}

We recall the definition of parallel composition (Definition 7) for closed networks $N=(X, \varphi, \pi)$ and $N^{\prime}=\left(X^{\prime}, \varphi^{\prime}, \pi^{\prime}\right)$. The network $N \otimes N^{\prime}$ is $\left(N \uplus N^{\prime}, \varphi^{\prime \prime}, \pi^{\prime \prime}\right)$ where:

- $\varphi^{\prime \prime}(x)=\varphi(x)$ for $x \in X$ and $\varphi^{\prime \prime}(y)=\varphi^{\prime}(y)$ for $y \in X^{\prime}$,

- $\pi^{\prime \prime}\langle x, i\rangle=\pi\langle x, i\rangle$ for $x \in X$ and $\pi^{\prime \prime}\langle y, i\rangle=\pi^{\prime}\langle y, i\rangle$ for $y \in X^{\prime}$.

For closed networks, parallel composition $N \otimes N^{\prime}$ and sequential composition $N \circ N^{\prime}$ agree. We also note that $N \otimes N^{\prime}$ is the coproduct of $N$ and $N^{\prime}$ in the category of networks and homomorphisms.

Definition 8. Let $x$ and $x^{\prime}$ be nodes in a network $N=(X, \varphi, \pi)$. They are directly connected, written $x \sim y$, if either $\pi\langle x, i\rangle=\left\langle x^{\prime}, j\right\rangle$ or $\pi\left\langle x^{\prime}, i\right\rangle=\langle x, j\rangle$, for some $i$ and $j$. Connectedness (of nodes) is the equivalence relation generated by $\sim$.

A non-empty equivalence class of nodes with respect to connectedness is called a connected component. A network is connected if any two of its nodes are connected, i.e., if it is itself a connected component.

In the following, we may refer to a network just by its set of nodes, leaving $\varphi$ and $\pi$ implicit. This convention is helpful as we are interested in decomposing a network into its connected components. We notice that a connected component is itself a (connected) network when equipped with the restrictions of $\varphi$ and $\pi$. Each network $X$ can be decomposed as:

$$
X \cong X_{1} \otimes \cdots \otimes X_{n}
$$

where the $X_{i}$ are the connected components of $X$.

We need some information on homomorphisms and connectedness. First, they clearly preserve connection, and so connectedness. Next: 
Lemma 2. Let $f: X \rightarrow Y$ be a homomorphism, and suppose that we have $f(x)=y \sim y^{\prime}$. Then there is an $x^{\prime}$ such that $x \sim x^{\prime}$ and $f\left(x^{\prime}\right)=y^{\prime}$.

We then have the following proposition:

Proposition 4. Let $f: X \rightarrow Y$ be a homomorphism. For each connected component $C$ of $X$, the image $f(C) \subseteq Y$ is a connected component of $Y$.

Corollary 1. Let $f: X \rightarrow Y$ be a homomorphism. If $Y$ is connected, then $f$ is a surjection.

The following immediate consequence will be important later.

Corollary 2. Let $f: X \rightarrow Y$ be a homomorphism and suppose that $Y$ is connected and $|X|=|Y|$. Then $f$ is an isomorphism.

Lemma 3. Let $f, g: X \rightarrow Y$ be homomorphisms. Suppose that $f(x)=g(x)$ and $x \sim x^{\prime}$. Then $f\left(x^{\prime}\right)=g\left(x^{\prime}\right)$.

This yields:

Proposition 5. Let $f, g: X \rightarrow Y$ be homomorphisms. If $X$ is connected and $f(x)=g(x)$ for some $x \in X$, then $f=g$.

The following upper bound on the number of homomorphisms is a direct consequence of this proposition.

Corollary 3. Let $X$ and $Y$ be networks, and suppose that $X$ is connected. Then $|\operatorname{hom}(X, Y)| \leq|Y|$.

Proposition 6. Let $f: X \rightarrow Y$ be a homomorphism. Then, for any $y \sim y^{\prime}$ in $Y,\left|f^{-1}(y)\right|=\left|f^{-1}\left(y^{\prime}\right)\right|$.

Proof. We may suppose, without loss of generality, that for some $i$ and $j$, $\pi_{Y}\langle y, i\rangle=\left\langle y^{\prime}, j\right\rangle$. Then we may define a bijection $\theta: f^{-1}(y) \cong f^{-1}\left(y^{\prime}\right)$ by $\theta(x)=\left(\pi_{X}\langle x, i\rangle\right)_{1}$; its inverse is given by $\theta^{-1}\left(x^{\prime}\right)=\left(\pi_{X}^{-1}\left\langle x^{\prime}, j\right\rangle\right)_{1}$.

The following corollary is then immediate:

Corollary 4. If $f: X \rightarrow Y$ is a homomorphism and $Y$ is connected, then $|X|$ is a multiple of $|Y|$.

\subsection{Interpretations over a Field $k$}

An interpretation $\mu$ of a (one-sorted) signature over a field $k$ is specified by a vector space $V^{\mu}$ and a linear map $\llbracket f \rrbracket^{\mu}: \llbracket a r_{\text {in }}(f) \rrbracket^{\mu} \rightarrow \llbracket a r_{\text {out }}(f) \rrbracket^{\mu}$ for each function symbol $f$, where $\llbracket m \rrbracket^{\mu}=\underbrace{V^{\mu} \otimes \cdots \otimes V^{\mu}}_{m}$. Let $X$ be a closed network over this signature, possibly empty or with trivial cycles. Its value with respect to the interpretation $\mu$ is then the trace of:

$$
\bigotimes_{x \in X} \llbracket a r_{\text {out }}(\varphi(x)) \rrbracket^{\mu} \stackrel{\hat{\pi}}{\longrightarrow} \bigotimes_{x \in X} \llbracket a r_{\text {in }}(\varphi(x)) \rrbracket^{\mu} \stackrel{\bigotimes_{x \in X} \llbracket \varphi(x) \rrbracket^{\mu}}{\longrightarrow} \bigotimes_{x \in X} \llbracket a r_{\text {out }}(\varphi(x)) \rrbracket^{\mu}
$$


where $\hat{\pi}$ is the linear map induced by $\pi$, and for $\bullet$ we put $\llbracket \bullet \rrbracket^{\mu}=\operatorname{dim} V^{\mu}$.

Note that for any two closed networks $X, Y$ over this signature we have that $\llbracket X \otimes Y \rrbracket^{\mu}=\llbracket X \rrbracket^{\mu} \llbracket Y \rrbracket^{\mu}$. It follows that the value of a network $X$ with $t$ trivial cycles and non-trivial connected components $X_{1}, \ldots, X_{n}$ is given by:

$$
\llbracket X \rrbracket^{\mu}=d^{t} \llbracket X_{1} \rrbracket^{\mu} \cdots \llbracket X_{n} \rrbracket^{\mu}
$$

where $d$ is the dimension of the interpretation of the sort by $\mu$.

Definition 9. Let $\mu_{1}, \mu_{2}$ be two interpretations. The interpretation $\mu_{1}+\mu_{2}$ is defined by:

$$
\begin{aligned}
& -V^{\mu_{1}+\mu_{2}}=V^{\mu_{1}} \oplus V^{\mu_{2}}, \\
& -\llbracket f \rrbracket^{\mu_{1}+\mu_{2}}\left(\bigotimes_{1 \leq i \leq a r_{i n}(f)} v_{i}+u_{i}\right)=\llbracket f \rrbracket^{\mu_{1}}\left(\bigotimes_{1 \leq i \leq a r_{i n}(f)} v_{i}\right)+\llbracket f \rrbracket^{\mu_{2}}\left(\bigotimes_{1 \leq i \leq a r_{i n}(f)}^{\bigotimes} u_{i}\right)
\end{aligned}
$$

where the evident inclusions of $\llbracket m \rrbracket^{\mu_{1}}$ and $\llbracket m \rrbracket^{\mu_{2}}$ in $\llbracket m \rrbracket^{\mu_{1}+\mu_{2}}$ have been omitted.

Proposition 7. Let $\mu_{1}, \mu_{2}$ be two interpretations. If $X$ is a connected network, then $\llbracket X \rrbracket^{\mu_{1}+\mu_{2}}=\llbracket X \rrbracket^{\mu_{1}}+\llbracket X \rrbracket^{\mu_{2}}$.

Proof. Let

$$
m: \bigotimes_{x \in X} \bigotimes_{1 \leq j \leq a r_{\text {out }}(\varphi(x))} V^{\mu_{1}} \oplus V^{\mu_{2}} \longrightarrow \bigotimes_{x \in X} \bigotimes_{1 \leq j \leq a r_{\text {out }}(\varphi(x))} V^{\mu_{1}} \oplus V^{\mu_{2}}
$$

be the linear map whose trace determines the value of $X$ under $\mu_{1}+\mu_{2}$. Also, let $m_{1}, m_{2}$ be the maps whose trace determines the value of $X$ under $\mu_{1}$ and $\mu_{2}$ respectively. Suppose that $v=\bigotimes_{x \in X} \bigotimes_{1 \leq j \leq a r_{\text {out }}(\varphi(x))} v_{\langle x, j\rangle}$ is a basis vector such that $\langle v \mid m(v)\rangle \neq 0$. Since $v$ is assumed to be a basis vector, we have that each $v_{\langle x, j\rangle}$ is either in $V^{\mu_{1}}$ or $V^{\mu_{2}}$, and is a basis vector of the respective space. We claim that all the $v_{\langle x, j\rangle}$ must lie in the same space. First, we notice that for given $x$ all the $v_{\pi_{X}^{-1}\langle x, i\rangle}$ for $i<a r_{i n} x$ must lie in the same space, for otherwise $\llbracket \varphi(x) \rrbracket^{\mu_{1}+\mu_{2}}\left(\bigotimes_{i} v_{\langle x, i\rangle}\right)=0$ and hence $m(v)=0$. Thus each $x$ is associated to either $V^{\mu_{1}}$ or $V^{\mu_{2}}$, and its directly connected nodes are also associated to the same space. Hence either $v \in V^{\mu_{1}}$ and $m(v)=m_{1}(v)$ or $v \in V^{\mu_{2}}$ and $m(v)=m_{2}(v)$. As the trace of $m$ is obtained by summing up all such $\langle v \mid m(v)\rangle$, we have the required result.

\subsection{The Counting Interpretation}

Let us fix a field $k$. We now describe the key part of the proof: given a connected network $X$ we define an interpretation $\mu(X, \lambda)$ over $k$ which, in essence, counts the contribution of each function symbol in the network $X$.

Definition 10. Let $X$ be a connected network and $\lambda \in k \backslash\{0\}$ be a non-zero scalar. The interpretation $\mu(X, \lambda)$ is defined as follows: 
- The (unique) sort 1 is interpreted as the vector space $V^{\mu(X, \lambda)}$ with basis the input ports of $X$, i.e., the set $\left\{\langle x, i\rangle \mid 1 \leq i \leq a r_{i n}(\varphi(x))\right\}$. (Hence $\operatorname{dim} V^{\mu(X, \lambda)}=\sum_{x \in X} a r_{i n}(\varphi(x))$.)

$-\llbracket f \rrbracket^{\mu(X, \lambda)}: \llbracket a r_{i n}(f) \rrbracket^{\mu(X, \lambda)} \rightarrow \llbracket a r_{\text {out }}(f) \rrbracket^{\mu(X, \lambda)}$ is given by:

$$
\llbracket f \rrbracket^{\mu(X, \lambda)}\left(\bigotimes_{1 \leq i \leq a r_{i n}(f)} p_{i}\right)=\lambda \sum_{\substack{x: \varphi(x)=f \\ p_{i}=\langle x, i\rangle}} \bigotimes_{1 \leq j \leq a r_{\text {out }}(f)} \pi\langle x, j\rangle
$$

Notice that if $\operatorname{ar}_{i n}(f)>0$ then the sum consists of at most one summand. In this case we have:

$$
\llbracket f \rrbracket^{\mu(X, \lambda)}\left(\bigotimes_{i} p_{i}\right)= \begin{cases}\lambda \bigotimes_{j} \pi\langle x, j\rangle & \text { if } p_{i}=\langle x, i\rangle \text { for all } i \\ 0 & \text { otherwise }\end{cases}
$$

That is to say, $\llbracket f \rrbracket^{\mu(X, \lambda)}$ is non-zero if it is applied to the input of an $f$-labelled node in $X$ and in this case returns the output of that node. The semantics of an input-less function symbol (a constant) is $\lambda$ times the sum over all its outputs occurring in $X$. We also notice that all function symbols that do not actually occur in $X$ receive zero meaning. If $F$ contains a symbol $f$ with $a r_{i n}(f)=$ $\operatorname{ar}_{\text {out }}(f)=0$ then, since $X$ is connected, either $X$ does not contain $f$-labelled nodes at all, hence $\llbracket f \rrbracket^{\mu(X, \lambda)}=0$, or $X$ consists of a single $f$-labelled node, in which case $V^{\mu}=k$ and $\llbracket f \rrbracket^{\mu(X, \lambda)}=\lambda$.

Theorem 1. Let $X$ and $Y$ be networks, and assume that $X$ is connected. Then, for any $\lambda \in k \backslash\{0\}$, we have:

$$
\llbracket Y \rrbracket^{\mu(X, \lambda)}=\lambda^{|Y|}|\operatorname{hom}(Y, X)|
$$

Proof. Recall that $V^{\mu(X, \lambda)}$ is the vector space with basis vectors the input ports of $X$, i.e., the set $\left\{\langle x, i\rangle \mid 1 \leq i \leq \operatorname{ar}_{i n}(\varphi(x))\right\}$. Let

$$
m: \bigotimes_{y \in Y} \bigotimes_{1 \leq j \leq a r_{\text {out }}(\varphi(y))} V^{\mu(X, \lambda)} \longrightarrow \bigotimes_{y \in Y} \bigotimes_{1 \leq j \leq a r_{\text {out }}(\varphi(y))} V^{\mu(X, \lambda)}
$$

be the linear map so that $\llbracket Y \rrbracket^{\mu(X, \lambda)}=\operatorname{Tr}(m)$. Unfolding the definition yields:

$$
m\left(\bigotimes_{y \in Y} \bigotimes_{1 \leq j \leq a r_{\text {out }}(\varphi(y))}\left\langle x_{(y, j)}, i_{(y, j)}\right\rangle\right)=\lambda^{|Y|} \bigotimes_{y \in Y} \sum_{x} \bigotimes_{1 \leq j \leq a r_{\text {out }}(\varphi(y))} \pi_{X}\langle x, j\rangle
$$

where the sum ranges over those $x \in X$ satisfying $\varphi_{X}(x)=\varphi_{Y}(y)$ and also $\left\langle x_{\pi_{Y}\langle y, i\rangle}, i_{\pi_{Y}\langle y, i\rangle}\right\rangle=\langle x, i\rangle$ for all $1 \leq i \leq \operatorname{ar}_{i n}\left(\varphi_{Y}(y)\right)$.

Now the trace of $m$ equals $\lambda^{|Y|}$ times the number of the basis vectors $v$ of the space $\bigotimes_{y \in Y} \bigotimes_{1 \leq j \leq a r_{o u t}(\varphi(y))} V^{\mu(X, \lambda)}$ which occur in $m(v)$, i.e., for which $\langle v \mid m(v)\rangle=\lambda^{|Y|}$. We show that these basis vectors are in 1-1 correspondence with homomorphisms from $Y$ to $X$. If $v=\bigotimes_{y \in Y} \bigotimes_{1 \leq j \leq a r_{\text {out }}(\varphi(y))}\left\langle x_{(y, j)}, i_{(y, j)}\right\rangle$ 
satisfies $\langle v \mid m(v)\rangle \neq 0$ then for each $y$ the sum in $m(v)$ must contain a summand corresponding to $v$. More precisely:

$$
\begin{aligned}
& \forall y \in Y \exists x \in X \\
& \varphi_{Y}(y)=\varphi_{X}(x) \\
& \forall i\left\langle x_{\pi_{Y}^{-1}\langle y, i\rangle}, i_{\pi_{Y}^{-1}\langle y, i\rangle}\right\rangle=\langle x, i\rangle(b) \\
& \forall j\left\langle x_{\langle y, j\rangle}, i_{\langle y, j\rangle}\right\rangle=\pi\langle x, j\rangle
\end{aligned}
$$

As explained above, either $X$ is a singleton set or it does not contain function symbols with neither inputs nor outputs. In each case, we have that for each $y \in Y$ there exists a unique $x \in X$ satisfying (b) and (c). In the former case, there is only one $x$ anyway; in the latter case either (b) or (c) is a nonempty conjunction and establishes uniqueness.

We have thus determined a function $f: Y \rightarrow X$ such that (b) and (c) hold with $x$ replaced with $f(y)$. We claim that $f$ is a homomorphism. Indeed, if $\pi_{Y}^{-1}\langle y, i\rangle=\left\langle y^{\prime}, j\right\rangle$ then by (b) we have $\langle f(y), i\rangle=\left\langle x_{\left\langle y^{\prime}, j\right\rangle}, i_{\left\langle y^{\prime}, j\right\rangle}\right\rangle$. On the other hand, (c) shows $\left\langle x_{\left\langle y^{\prime}, j\right\rangle}, i_{\left\langle y^{\prime}, j\right\rangle}\right\rangle=\pi_{X}\left\langle f\left(y^{\prime}\right), j\right\rangle$, thus $\langle f(y), i\rangle=\pi_{X}\left\langle f\left(y^{\prime}\right), j\right\rangle$ or $\pi_{X}^{-1}\langle f(y), i\rangle=\left\langle f\left(y^{\prime}\right), j\right\rangle$ establishing homomorphism.

Conversely, if $f: Y \rightarrow X$ is a homomorphism, we define a basis vector $v=$ $\bigotimes_{y \in Y} \bigotimes_{1 \leq j \leq a r_{o u t}(\varphi(y))}\left\langle x_{\langle y, j\rangle}, i_{\langle y, j\rangle}\right\rangle$ by:

$$
\left\{\begin{array}{l}
x_{\langle y, j\rangle}=f\left(y^{\prime}\right) \\
i_{\langle y, j\rangle}=i
\end{array} \quad \text { when } \pi_{Y}\langle y, j\rangle=\left\langle y^{\prime}, i\right\rangle\right.
$$

Now, towards showing (a), (b), (c) above, given $y \in Y$ we put $x=f(y)$. Condition (a) follows directly from the homomorphism property; condition (b) is direct from the definition of $\left\langle x_{\langle y, j\rangle}, i_{\langle y, j\rangle}\right\rangle$; for condition (c), we assume $\pi_{Y}\langle y, j\rangle=\left\langle y^{\prime}, i\right\rangle$ hence $\pi_{X}\langle f(y), j\rangle=\left\langle f\left(y^{\prime}\right), i\right\rangle=\left\langle x_{\langle y, j\rangle}, i_{\langle y, j\rangle}\right\rangle$ using the homomorphism property and the definition of $\left\langle x_{\langle y, j\rangle}, i_{\langle y, j\rangle}\right\rangle$.

It is obvious that going back and forth starting with a homomorphism $f$ yields that homomorphism back. To show the converse, assume that we are given a basic vector determined by a family $\left\{\left\langle\hat{x}_{\langle y, j\rangle}, \hat{i}_{\langle y, j\rangle}\right\rangle\right\}_{\langle y, j\rangle}$. We define a homomorphism $f: Y \rightarrow X$ by letting $f(y)$ be the unique $x$ satisfying conditions (a), (b), (c) above. We then define another basic vector $\left\{\left\langle x_{\langle y, j\rangle}, i_{\langle y, j\rangle}\right\rangle\right\}_{\langle y, j\rangle}$ by (11).

Given $y \in Y$ and $1 \leq j \leq \operatorname{ar}_{\text {out }}(\varphi(y))$ we have:

$$
\left\langle\hat{x}_{\langle y, j\rangle}, \hat{i}_{\langle y, j\rangle}\right\rangle=\pi_{X}\langle f(y), j\rangle
$$

by condition (b) above. On the other hand, if $\pi_{Y}\langle y, j\rangle=\left\langle y^{\prime}, i\right\rangle$ then:

$$
\pi_{X}\langle f(y), j\rangle=\left\langle f\left(y^{\prime}\right), i\right\rangle=\left\langle x_{\langle y, j\rangle}, i_{\langle y, j\rangle}\right\rangle
$$

by the homomorphism property and (11), thus:

$$
\left\langle\hat{x}_{\langle y, j\rangle}, \hat{i}_{\langle y, j\rangle}\right\rangle=\left\langle x_{\langle y, j\rangle}, i_{\langle y, j\rangle}\right\rangle
$$

as required. 
Theorem 1 and Proposition 7 immediately yield:

Theorem 2. Let $X$ be a network with connected components $X_{1}, \ldots, X_{n}$, let $\lambda_{1}$, $\ldots, \lambda_{n}$ be non-zero scalars, and let $Y$ be a network with connected components $Y_{1}, \ldots, Y_{m}$. Then we have:

$$
\llbracket Y \rrbracket^{\sum_{i=1}^{n} \mu\left(X_{i}, \lambda_{i}\right)}=\prod_{j=1}^{m} \sum_{i=1}^{n} \lambda_{i}^{\left|Y_{j}\right|}\left|\operatorname{hom}\left(Y_{j}, X_{i}\right)\right|
$$

\section{Completeness Results}

We begin by considering closed networks over a one-sorted signature. In the following definition we assume a standard enumeration of (the isomorphism classes) of the connected non-empty and non-trivial such networks.

Definition 11. Let $X$ be a closed network over a one-sorted signature, and suppose its non-trivial connected components are $X_{1}, \ldots, X_{n}(n \geq 0)$, and let $\lambda_{1}, \ldots, \lambda_{n}$ be distinct variables (taken from some standard enumeration of variables). Then the interpretation $\mu_{X}$ over $\mathbb{Q}\left(\lambda_{1}, \ldots, \lambda_{n}\right)$ is given by:

$$
\mu_{X}=\mu\left(X_{1}, \lambda_{1}\right)+\cdots+\mu\left(X_{n}, \lambda_{n}\right)+\zeta_{2}
$$

where $\zeta_{2}$ is the interpretation interpreting 1 by a two-dimensional space and assigning all function symbols the value 0 .

Now if $Y$ is any closed network over the same signature as $X$, with non-trivial connected components $Y_{1}, \ldots, Y_{m}$ and with $t$ trivial cycles, we have by the above remarks on the interpretation of such networks, Proposition 17 and Theorem 2 that:

$$
\llbracket Y \rrbracket^{\mu_{X}}=d^{t} \prod_{j=1}^{m} \sum_{i=1}^{n} \lambda_{i}^{\left|Y_{j}\right|}\left|\operatorname{hom}\left(Y_{j}, X_{i}\right)\right|
$$

where $d \geq 2$ is the dimension of the interpretation of 1 by $\mu_{X}$. Note that this is a polynomial in $\lambda_{1}, \ldots, \lambda_{n}$ with positive integer coefficients, and non-zero in case $n>0$ and $X$ and $Y$ have the same non-trivial connected components up to isomorphism. Writing $\operatorname{deg}\left(\lambda_{i}, \llbracket Y \rrbracket^{\mu_{X}}\right)$ for the largest exponent of $\lambda_{i}$ in $\llbracket Y \rrbracket^{\mu_{X}}$, we have:

$$
\operatorname{deg}\left(\lambda_{i}, \llbracket Y \rrbracket^{\mu_{X}}\right)=\sum_{j: \operatorname{hom}\left(Y_{j}, X_{i}\right) \neq \emptyset}\left|Y_{j}\right|
$$

where $Y_{1}, \ldots, Y_{m}$ are the components of $Y$.

Lemma 4. Let $X$ and $Y$ be closed networks over a one-sorted signature, at least one of which has a non-trivial connected component. If

$$
\llbracket X \rrbracket^{\mu_{X}}=\llbracket Y \rrbracket^{\mu_{X}}
$$


and

$$
\llbracket X \rrbracket^{\mu_{Y}}=\llbracket Y \rrbracket^{\mu_{Y}}
$$

then $X$ and $Y$ are isomorphic.

Proof. Let $X_{1}, \ldots, X_{m}$ and $Y_{1}, \ldots, Y_{n}$ be the standard enumerations of the nontrivial connected components of $X$ and $Y$, respectively.

Let $U$ be a connected component in $X$ or $Y$. The height of $U$ is defined as the length of the longest sequence of homomorphisms

$$
U_{0} \stackrel{f_{0}}{\longrightarrow} U_{1} \stackrel{f_{1}}{\longrightarrow} U_{2} \longrightarrow \ldots \stackrel{f_{k-1}}{\longrightarrow} U_{k}=U
$$

where $U_{i}$ are connected components in $X$ or $Y$, and none of the $f_{i}$ are isomorphisms. Notice that the height is well-defined by Corollaries 1 and 2.

The multiplicity of component $U$ in $X$ (or $Y$ ) is defined as the number of isomorphic copies of $U$ in $X$ (or $Y$ ). We show by course-of-values induction on $h$ that each component of $X$ or $Y$ of height $h$ has the same multiplicity in $X$ and in $Y$.

So assume that $U$ is a connected component of height $h$ and that components of height less than $h$ have equal multiplicities in $X$ and $Y$. Let us write $x$ and $y$ for the multiplicity of $U$ in $X$ and $Y$ respectively. By the definition of height we have:

$$
\sum_{i: \operatorname{hom}\left(X_{i}, U\right) \neq \emptyset}\left|X_{i}\right|=x|U|+\sum_{i: \operatorname{hom}\left(X_{i}, U\right) \neq \emptyset \wedge \operatorname{height}\left(X_{i}\right)<h}\left|X_{i}\right|
$$

and:

$$
\sum_{j: \operatorname{hom}\left(Y_{j}, U\right) \neq \emptyset}\left|Y_{i}\right|=y|U|+\sum_{i: \operatorname{hom}\left(Y_{i}, U\right) \neq \emptyset \wedge \operatorname{height}\left(Y_{i}\right)<h}\left|Y_{i}\right|
$$

Now, supposing without loss of generality that $U$ occurs in $X$ as $X_{1}$, we conclude by equation 3 that:

$$
\sum_{i: \operatorname{hom}\left(X_{i}, U\right) \neq \emptyset}\left|X_{i}\right|=\operatorname{deg}\left(\lambda_{1}, \llbracket X \rrbracket^{\mu_{X}}\right)=\operatorname{deg}\left(\lambda_{1}, \llbracket Y \rrbracket^{\mu_{X}}\right)=\sum_{j: \operatorname{hom}\left(Y_{j}, U\right) \neq \emptyset}\left|Y_{i}\right|
$$

Combining this with the induction hypothesis shows $x|U|=y|U|$, hence $x=y$.

So $X$ and $Y$ have the same non-trivial connected components, up to isomorphism. So, as $\llbracket X \rrbracket^{\mu_{X}}=\llbracket Y \rrbracket^{\mu_{X}}$, we see by equation 2 above and the following remark that they have the same number of trivial cycles, which concludes the proof.

Theorem 3. If two networks over a given signature have equal value under all interpretations over fields of the form $\mathbb{Q}\left(\lambda_{1}, \ldots, \lambda_{n}\right)$ then they are isomorphic.

Proof. We have already described how the general case can be reduced in turn to that of one-sorted signatures and then to that of closed such networks. The previous lemma deals with all such cases except the trivial one where both networks consist only of trivial cycles. 
In order to strengthen our completeness result to fields of characteristic 0, we encode polynomials with positive integer coefficients as natural numbers:

Proposition 8. Let $d$ and $C$ be positive integers. There exist natural numbers $k_{1}, \ldots, k_{n}$ such that for any polynomials $p, q \in \mathbb{N}\left[\lambda_{1}, \ldots, \lambda_{n}\right]$ with total degree less or equal to $d$ and coefficients smaller than $C$ we have:

$$
p=q \Longleftrightarrow p\left(k_{1}, \ldots, k_{n}\right)=q\left(k_{1}, \ldots, k_{n}\right)
$$

We then have:

Theorem 4. Let $k$ be a field of characteristic 0. If two networks over a given signature have equal value under all interpretations over $k$ then they are isomorphic.

A number of natural questions arise on considering this theorem. As regards generalisations, we do not know if the corresponding result is true for any field of positive characteristic. Nevertheless, a small refinement of our proof yields the following weaker result:

Theorem 5. If two networks have equal value under all interpretations over all finite fields then they are isomorphic.

Proof. For this, one makes use of the fact that for positive integers $d, C$ there always exists a finite field $k$ and $l_{1}, \ldots, l_{n} \in k$ such that for any two polynomials $p, q \in \mathbb{N}\left[\lambda_{1}, \ldots, \lambda_{n}\right]$ with total degree less or equal to $d$ and coefficients smaller than $C$ one has $p=q$ iff $p\left(l_{1}, \ldots, l_{n}\right)=q\left(l_{1}, \ldots, l_{n}\right)$ in $k$, and then simply proceeds as in the proof of Theorem 4, taking a finite field with characteristic large enough so that no undesired cancellations occur.

One may also ask if Theorem 4 can be strengthened. Perhaps there is a uniform bound on the dimensions of the vector spaces needed for completeness. Alternatively there may be a result similar to those of Statman for the simply typed $\lambda$-calculus [13. This might associate to each network $N$ a bound on the dimensions of the vector spaces needed to decide whether any other network is isomorphic to $N$; there may even a be single interpretation such that another network is isomorphism to $N$ iff it has the same value as $N$ in that interpretation.

\subsection{Completeness for Compact Closed Categories}

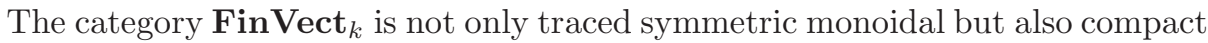
closed. So it is natural to ask if our completeness result also holds for compact closed categories. This is indeed the case: it is a corollary of the result for the traced case and the structure theorem of Joyal, Street and Verity [6].

As noted before, every compact closed category has a unique trace. The structure theorem says that the forgetful 2-functor from the 2-category CompCat of compact closed categories to the 2-category TrMon of traced symmetric monoidal categories has a left biadjoint whose unit is fully faithful. More concretely, given a traced symmetric monoidal category $\mathcal{C}$, there is a compact 
closed category Int $\mathcal{C}$ whose objects are pairs of objects of $\mathcal{C}$ and whose arrows from $\left(A^{+}, A^{-}\right)$to $\left(B^{+}, B^{-}\right)$are the arrows from $A^{+} \otimes B^{-}$ro $B^{+} \otimes A^{-}$ in $\mathcal{C}$. The identity arrow on $\left(A^{+}, A^{-}\right)$is $i d_{A^{+} \otimes A^{-}}$, and the composition of $f:\left(A^{+}, A^{-}\right) \rightarrow\left(B^{+}, B^{-}\right)$with $g:\left(B^{+}, B^{-}\right) \rightarrow\left(C^{+}, C^{-}\right)$is given by:

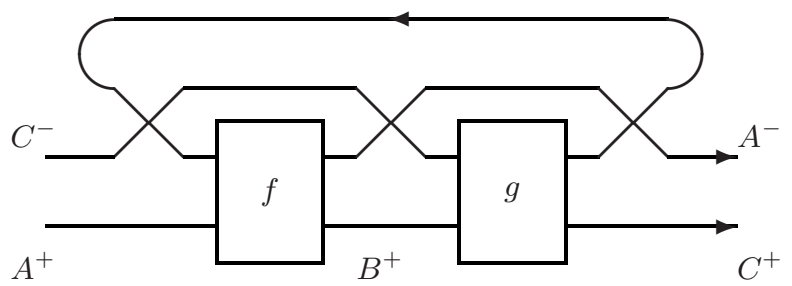

As regards the compact closed structure, the interested reader is referred to [6] (but our symmetric case is much simpler than the original braided case). In the case $\boldsymbol{C}=\operatorname{Net}_{(S, F)}$, we regard $\mathbf{I n t} \mathbf{N e t}_{(S, F)}$ as a category of 'bi-directional networks' modulo isomorphism, where a bi-directional network from $\left(v^{+}, v^{-}\right)$to $\left(w^{+}, w^{-}\right)$is just a network from $v^{+} w^{-}$to $w^{+} v^{-}$.

There is a fully faithful strong traced functor $\mathcal{N}: \mathcal{C} \rightarrow \operatorname{Int} \mathcal{C}$ sending $A$ to $(A, I)$. Furthermore, for any compact closed category $\mathcal{D}$ and any strong traced functor $\mathcal{F}: \mathcal{C} \rightarrow \mathcal{D}$, there is a unique (up to isomorphism) strong monoidal functor $\overline{\mathcal{F}}:$ Int $\mathcal{C} \rightarrow \mathcal{D}$ such that $\overline{\mathcal{F}} \circ \mathcal{N}$ is isomorphic to $\mathcal{F}$; explicitly, $\overline{\mathcal{F}}$ sends $\left(A^{+}, A^{-}\right)$ to $\mathcal{F} A^{+} \otimes\left(\mathcal{F} A^{-}\right)^{*}$, and $f:\left(A^{+}, A^{-}\right) \rightarrow\left(B^{+}, B^{-}\right)$to:

$$
\begin{aligned}
& \mathcal{F} A^{+} \otimes\left(\mathcal{F} A^{-}\right)^{*} \stackrel{i d \otimes \eta \otimes i d}{\longrightarrow} \mathcal{F} A^{+} \otimes \mathcal{F} B^{-} \otimes\left(\mathcal{F} B^{-}\right)^{*} \otimes\left(\mathcal{F} A^{-}\right)^{*} \\
& \stackrel{\simeq}{\longrightarrow} \mathcal{F}\left(A^{+} \otimes B^{-}\right) \otimes\left(\mathcal{F} B^{-}\right)^{*} \otimes\left(\mathcal{F} A^{-}\right)^{*} \\
& \stackrel{(\mathcal{F} f) \otimes i d}{\longrightarrow} \mathcal{F}\left(B^{+} \otimes A^{-}\right) \otimes\left(\mathcal{F} B^{-}\right)^{*} \otimes\left(\mathcal{F} A^{-}\right)^{*} \\
& \stackrel{\simeq}{\longrightarrow} \mathcal{F} B^{+} \otimes\left(\mathcal{F} B^{-}\right)^{*} \otimes\left(\mathcal{F} A^{-}\right)^{*} \otimes \mathcal{F} A^{-} \\
& \stackrel{i d \otimes \varepsilon}{\longrightarrow} \mathcal{F} B^{+} \otimes\left(\mathcal{F} B^{-}\right)^{*}
\end{aligned}
$$

In particular, $\operatorname{TrMon}(\mathcal{C}, \mathcal{D})$ is equivalent to $\operatorname{CompCat}(\operatorname{Int} \mathcal{C}, \mathcal{D})$.

We can routinely define the notion of interpretations of signatures in a compact closed category $\mathcal{D}$ and morphisms between them, but this is the same as giving interpretations of signatures in $\mathcal{D}$ regarded as a traced monoidal category and morphisms between them. From this, we note that Int $\mathbf{N e t}_{(S, F)}$ is the free compact closed category over the signature $(S, F)$ because, for any compact closed $\mathcal{D}$, we have the following equivalences:

$$
\operatorname{Mod}((S, F), \mathcal{D}) \simeq \operatorname{TrMon}\left(\operatorname{Net}_{(S, F)}, \mathcal{D}\right) \simeq \operatorname{CompCat}\left(\operatorname{Int} \operatorname{Net}_{(S, F)}, \mathcal{D}\right)
$$

So we can speak of the value of a bidirectional net given an interpretation of its signature over $k$, i.e., in $\mathbf{F i n V e c t}_{k}$ : one simply applies the functor obtained from the interpretation by the above chain of equivalences to the isomorphism class of the net.

Corollary 5. Let $k$ be a field of characteristic 0 . If two bidirectional nets have equal value under all interpretations over $k$ then they are isomorphic. 
For the proof, one uses the definition of $\overline{\mathcal{F}}$ to reduce the question to the case of $\operatorname{Net}_{(S, F)}$ and the result is then immediate from Theorem 4 .

\section{Acknowledgements}

Hasegawa acknowledges the support of the Japanese Ministry of Education, Culture, Sports, Science and Technology, Grant-in-Aid for Young Scientists (B) 17700013. Plotkin acknowledges the support of a Royal Society-Wolfson award.

\section{References}

1. Bloom, S., Ésik, Z.: Iteration Theories, EATCS Monographs on Theoretical Computer Science. Springer, Heidelberg (1993)

2. Hasegawa, M.: Recursion from cyclic sharing: traced monoidal categories and models of cyclic lambda calculi. In: de Groote, P., Hindley, J.R. (eds.) TLCA 1997. LNCS, vol. 1210, pp. 196-213. Springer, Heidelberg (1997)

3. Hasegawa, M.: Models of Sharing Graphs: A Categorical Semantics of let and letrec. Distinguished Dissertation Series. Springer, Heidelberg (1999), also available as Ph.D. thesis ECS-LFCS-97-360, University of Edinburgh (1997)

4. Hyland, M., Power, A.J.: Symmetric monoidal sketches and categories of wirings. Electr. Notes Theor. Comput. Sci 100, 31-46 (2004)

5. Joyal, A., Street, R.: Braided tensor categories. Adv. Math. 102, 20-78 (1993)

6. Joyal, A., Street, R., Verity, D.: Traced monoidal categories. Math. Proc. Cambridge Phil. Soc. 119(3), 447-468 (1996)

7. Mac Lane, S.: Categories for the Working Mathematician. Springer, Heidelberg (1971)

8. Pardo, D., Rabinovich, A.M., Trakhtenbrot, B.A.: Synchronous circuits over continuous time: feedback, reliability and completeness. Fundam. Inform. 62(1), 123-137 (2004)

9. Rabinovich, A.M., Trakhtenbrot, B.A.: Nets and data flow interpreters. In: Proc. Fourth Symp. on Logic in Computer Science, pp. 164-174. IEEE Computer Society Press, Washington (1989)

10. Simpson, A.K.: Categorical completeness results for the simply-typed lambdacalculus. In: Dezani-Ciancaglini, M., Plotkin, G. (eds.) TLCA 1995. LNCS, vol. 902, pp. 414-427. Springer, Heidelberg (1995)

11. Simpson, A.K., Plotkin, G.: Complete axioms for categorical fixed-point operators. In: Proc. Fifteenth Symp. on Logic in Computer Science, pp. 30-41. IEEE Computer Society Press, Washington (2000)

12. Soloviev, S.V.: Proof of a conjecture of S. Mac Lane. Ann. Pure Appl. Logic 90, 101-162 (1997)

13. Statman, R.: Completeness, invariance, and definability. J. Symbolic Logic 47, 17$26(1982)$

14. Ştefănescu, G.: Network Algebra. Series in Discrete Mathematics and Theoretical Computer Science. Springer, Heidelberg (2000)

15. Trakhtenbrot, B.A.: On operators, realizable in logical nets. Doklady AN SSSR (Proceedings of the USSR Academy of Sciences) 112(6), 1005-1007 (1957)

16. Trakhtenbrot, B.A.: On the power of compositional proofs for nets: relationships between completeness and modularity. Fundam. Inform. 30(1), 83-95 (1997)

17. Yetter, D.N.: Functorial Knot Theory. World Scientific, Singapore (2001) 